\title{
Review: antidepressants improve headache in patients with chronic headache
}

\author{
Tomkins GE, Jackson JL, O'Malley PG, et al. Treatment of chronic headache with antidepressants: a meta-analysis. Am J \\ Med 2001 Jul;111:54-63.
}

QUESTION: In patients with chronic headache, how effective are antidepressants?

\section{Data sources}

Studies were identified by searching Medline (1966 to December 1998), PsycLIT (1974 to December 1998), EMBASE/Excerpta Medica (1974 to December 1998), and the Cochrane Library. Bibliographies of relevant articles were reviewed, and Federal Research in Progress was searched for information on unpublished trials.

\section{Study selection}

Studies in any language were selected if they were randomised controlled trials (RCTs) comparing the effectiveness of antidepressants with placebo in patients with chronic headache and if measurable outcomes were reported.

\section{Data extraction}

Data were extracted on setting, country of origin, patient inclusion and exclusion criteria, sample size, demographical characteristics, comorbid psychiatric disease, concurrent use of analgesic medications, key components of the intervention, study quality, adverse effects, and outcomes. Outcomes were overall improvement in headache $(>50 \%$ reduction in Headache Index scores, global improvement based on $>50 \%$ improvement, impression of improvement, or headache frequency or severity) and effect size of treatment (measured in standard deviation units) on headache burden.

\section{Main results}

38 RCTs met the selection criteria. Interventions included tricylic antidepressants (TCAs) (19 RCTs), serotonin blocking agents (18 RCTs), and selective serotonin reuptake inhibitors (SSRIs) (7 RCTs). The rate of overall improvement in headache was greater in the treatment group than the placebo group $(\mathrm{p}<0.05)$ (table). Improvement in headache burden (measured by effect size of treatment) was greater in the treatment group than the placebo group (standardised mean difference [SMD] 0.94, 95\% CI 0.64 to 1.12 ). Consumption of analgesic medication (measured by effect size of treatment) was lower in the treatment group than the placebo group (SMD -0.7, CI -0.9 to -0.5). Outcomes did not differ by type of headache (migraine $v$ tension) or by category of antidepressant.

\section{Conclusion}

In patients with chronic headache, treatment with antidepressants is associated with overall improvement in headache and improvement in headache burden.

\section{COMMENTARY}

The meta-analysis by Tomkins et al concludes that 1 in 3 patients with chronic headaches benefits from antidepressants. The effect is independent of headache type (migraine or tension) and drug class (TCAs, serotonin receptor antagonists, or SSRIs), with the possible exception that SSRIs are not as well studied and may be less effective. The effect seems independent of the presence of depression, but studies are inconsistent.

The results of Tomkins $e t a l$ are consistent with those of other systematic reviews ${ }^{12}$ and indicate that antidepressants, especially TCAs, are a reasonable first choice to treat headaches. The choice of treatment depends on the patient's comorbid illnesses that might be improved or worsened by the treatment for headache.

The authors' thorough analysis shows problems in the published studies. First, the authors found statistically significant heterogeneity among the studies with a 4 -fold variation in rate ratios. This finding is not surprising given the range of drugs and patients. Second, the authors found statistical evidence of publication bias. Although unpublished studies will probably not reverse the results of this meta-analysis, unpublished negative studies will probably lessen the degree of benefit.

Two types of studies are now needed. First, we need larger studies to reduce the effect of publication bias and allow effective analysis of the true benefits and variation in results. Second, we need comparisons of antidepressants with other treatments and combinations of treatment. One study, published since the meta-analysis by Tomkins $e t$ al was written, meets these criteria: Holroyd et $a l^{3}$ compared TCAs with stress management treatment and placebo in 233 patients with tension headaches (almost twice the size of the largest trial in this meta-analysis). 38\% of patients benefited from TCAs (less than in the current analysis), whereas combined treatment benefited $64 \%$ of patients. ${ }^{3}$

$$
\begin{array}{r}
\text { Robert Badgett, MD } \\
\text { University of Texas Health Sciences Center } \\
\text { San Antonio, Texas, USA }
\end{array}
$$

1 Pryse-Phillips WE, Dodick DW, Edmeads JG, et al. Guidelines for the diagnosis and management of migraine in clinical practice. Canadian Headache Society. CMAJ 1997;156: practice. $1273-87$.

2 Redillas C, Solomon S. Prophylactic pharmacological treatment of chronic daily headache. Headache 2000;40:83102.

3 Holroyd KA, O'Donnell FJ, Stensland M, et al. Management of chronic tension-type headache with tricyclic antidepressant medication, stress management therapy, and their combination: a randomized controlled trial. JAMA 2001;285: 2208-15.

Antidepressants v placebo for chronic headache*

\begin{tabular}{lllll} 
& \multicolumn{2}{l}{ Weighted event ratest } & & \\
\cline { 2 - 3 } Outcome at 4 to 27 weeks & Antidepressants & Placebo & RBI (95\% Cl) & NNT (Cl) \\
Overall improvement & $65 \%$ & $33 \%$ & $100 \%(60$ to 140) & $4(3$ to 5$)$ \\
\hline
\end{tabular}

Source of funding: not stated.

For correspondence: Dr J L Jackson, Medicine-EDP Bethesda, MD, USA. jejackson@usuhs.mil. 